\title{
Adaptive Radiotherapy in Orbital Rhabdomyosarcoma: A Case Report
}

\author{
Authors
}

\section{Heena Rathod, Maitrik Mehta, Amit Kichloo, Sakina Mankada, Rajal Shah}

Department of Radiation Oncology, The Gujarat Cancer and Research Institute, Ahmedabad, Gujarat, India Corresponding Author

\section{Dr Heena Rathod}

Department of Radiation Oncology, The Gujarat Cancer and Research Institute, Ahmedabad, Gujarat, India

\begin{abstract}
Rhabdomyosarcoma is highly malignant soft tissue sarcoma having mesoderm or myotome derived skeletal muscle origin. It is most common soft tissue sarcoma of childhood and accounts for $4 \%$ of pediatric solid malignancy. Orbital RMS accounts for 9-10\% of head and neck RMSs. It has favorable prognosis with long survival. The treatment of orbital RMS has multidisciplinary approach. In this article, we have reported a case of 8 year old girl with orbital RMS in which we had planned adaptive radiotherapy due to excellent response of tumor to the chemoradiation.

Keywords: Orbital rhabdomyosarcoma, chemotherapy, Adaptive radiation, IMRT.
\end{abstract}

\section{Introduction}

Rhabdomyosarcoma (RMS) is highly malignant soft tissue sarcoma which arises from undifferentiated mesoderm or myotome derived skeletal muscle. It accounts for $4 \%$ of pediatric solid malignancy. It is most common childhood soft tissue sarcoma with male to female ratio is 1.5:1. At the time of diagnosis most patients are of less than 10 years of age. Most frequent sites of involvement are genitourinary, head and neck orbit, extremities, trunk and others. Most ocular RMS arises from soft tissue of orbit, but it can arise from ocular adnexal structures. Orbital RMS carries favorable prognosis. In this article, we have reported a case of 8 year old girl diagnosed with orbital RMS in which we had planned adaptive radiation therapy due to excellent response to chemoradiation.

\section{Case Report}

An 8 year girl presented to our institute with localized painless swelling on her left eye for 10 days. It was associated with proptosis and periorbital swelling. There was no complaint of fever or pus discharge from the left eye and no history of any ocular trauma. She was completely immunized for her age.

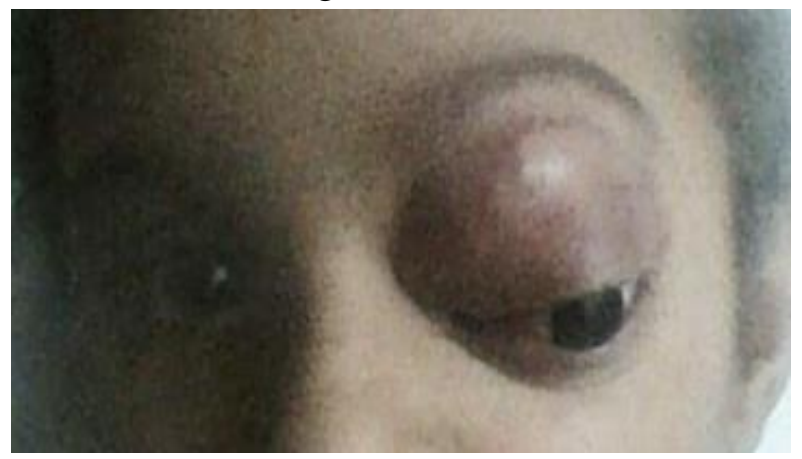

Fig.1 Patient at initial presentation 
On physical examination, vitals were normal. On local examination, there was $3 * 2 \mathrm{~cm}$ swelling of left upper eyelid just above the medial canthus. Vision in both eyes was 6/6. There were no clinically palpable neck nodes. Excisional biopsy of left eyelid was done under anesthesia, which was suggestive of malignant spindle cell tumor. Then IHC was done that showed vimentin, desmin, myo D1 - positive and myoglobin negative.

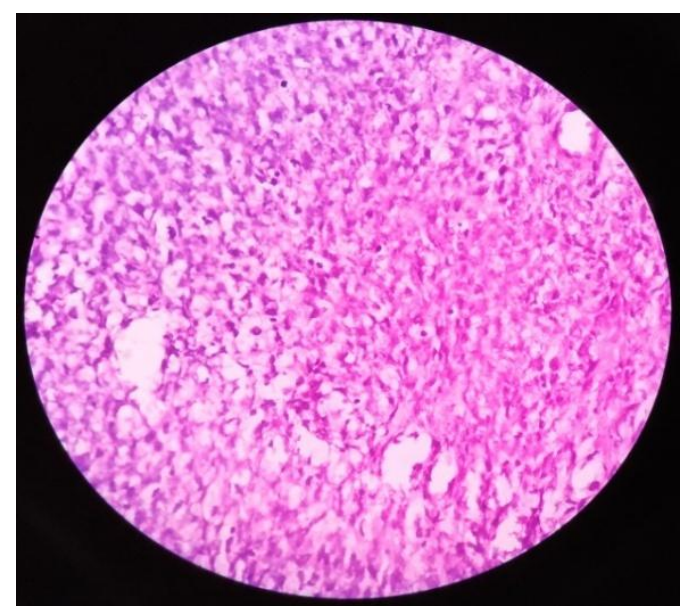

Fig.2 Slide showing embryonal variety of RMS

MRI of orbit showed $23 * 25 * 16 \mathrm{~mm}$ lesion in medial canthus of left eyelid extending posteriorly into intraconal compartment of left orbit. There was loss of fat plane with superior rectus and superior oblique muscle with external compression on orbital globe on posterior aspect. Lesion was abutting lacrimal gland. No metastasis found on further work up.

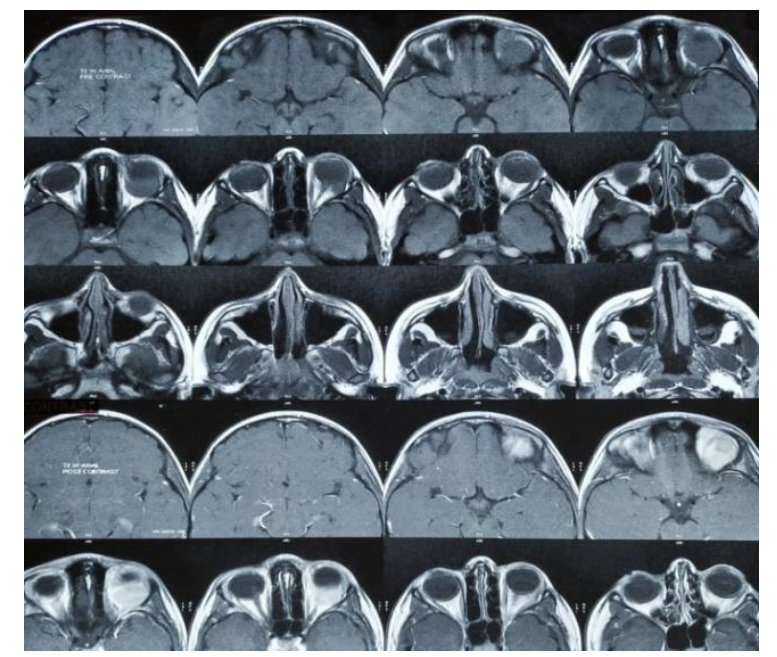

Fig.3 MRI showing lesion in left orbit
The patient was started on $\mathrm{V}+\mathrm{A}$ (Vincristine + Actinomycin D) chemotherapy protocol. After completion of 24 weeks of chemotherapy, Imaging of brain \& orbit was done, that showed $34 * 14 \mathrm{~mm}$ lesion involving roof of left orbit on medial aspect. Lesion abuts left lacrimal gland and left superior rectus with loss of fat plan.

Patient had received radiation therapy $45 \mathrm{~Gy} / 25 \#$ at $1.8 \mathrm{~Gy} / \#$ by conformal IMRT technique. During that, patient had shown excellent response to treatment in 11\#. So, adaptive radiation planning was done according to disease size. Then patient was given $25.2 \mathrm{~Gy} / 14$ \#. Patient had taken radiation therapy with concurrent vincristine till week 32.

Patient is on regular follow up. Last MRI orbit shows $18 * 10^{*} 6 \mathrm{~mm}$ lesion involving roof of left orbit on medial aspect. Lesion abuts left superior rectus and superior oblique muscles. Patient has shown excellent subjective response to chemoradiation. Vision is $6 / 6$ in both eyes at present. At present patient is kept under surveillance.

\section{Discussion}

Primary orbital RMS affects mainly young children. Locally invasive tumor has potential for local spread along fascial or muscle planes. In RMS, hematogenous metastases may present in lung, bone marrow and bone. Overall risk of lymph node spread is $15 \%$ but in orbital RMS lymphatic spread is rare. Orbital RMS most commonly found in soft tissue of orbit.

Clinical features of orbital RMS may include proptosis, eyelid edema, redness of eye, decreased vision. The most favored position of lesion is in the superonasal part of orbit that lead to proptosis with lateral displacement of the eyeball. Our patient presented with left sided periorbital swelling and proptosis with normal vision.

RMS may be associated with other developmental disorder or genetic disease. Orbital RMS can also occur as secondary tumor after radiation treatment for retinoblastoma or squamous cell carcinoma of 
eyelid. Our patient was normal child without any developmental disorder or genetic mutation or any history of previous radiation.

Diagnosis and management of orbital RMS is done with multidisciplinary approach. Imaging is important to know the extension of disease, staging, evaluation after chemotherapy. Imaging may include contrast enhanced CT scan or MRI of local part. Orbital RMS usually occurs in extraconal compartment of orbitbut it can also extend intraconally. CT scan better shows bone erosion or involvement. MRI is important for soft tissue involvement perineural invasion and intracranial spread. In our patient, we had done MRI which showed tumor was in intraconal compartment of left orbit.

The diagnosis is confirmed by incisional or excisional biopsy. The histopathological subtypes of RMS include embryonal, alveolar, pleomorphic \& undifferentiated. Embryonal RMS is very common and carry favourable prognosis. Most of the orbital RMSs are of embryonal variety, only $10 \%$ accounts for alveolar subtype. IHC studies can be done with marker like actin, desmin, myoD1, myoglobin and myosin. In our patient, excisional biopsy was done which showed embryonal variety of orbital RMS with vimentin, desmin and myo D1 positive and myoglobin negative.

Following biopsy, staging is done according to IRS post surgical staging system.

\begin{tabular}{|l|l|}
\hline Group I & Localized disease, completely resected \\
\hline & A: Confined to organ or muscle of origin \\
\hline & $\begin{array}{l}\text { B: Infiltration outside organ or muscle of origin; } \\
\text { regional nodes not involved }\end{array}$ \\
\hline Group II & Compromised or regional resection \\
\hline & $\begin{array}{l}\text { A: Grossly resected tumor with microscopic } \\
\text { residual disease }\end{array}$ \\
\hline $\begin{array}{l}\text { B: Regional disease, completely resected, in } \\
\text { tumor into adjacent organ may exist }\end{array}$ \\
\hline $\begin{array}{l}\text { C: Regional disease with involved nodes, grossly } \\
\text { resected, but with evidence of microscopic } \\
\text { residual disease }\end{array}$ \\
\hline III & $\begin{array}{l}\text { Incomplete resection or biopsy with gross } \\
\text { residual disease }\end{array}$ \\
\hline $\begin{array}{l}\text { Group } \\
\text { IV }\end{array}$ & \begin{tabular}{l} 
Distant metastases at diagnosis \\
\hline
\end{tabular}
\end{tabular}

Based on the staging treatment modality is decided that include chemotherapy, radiotherapy and surgery. Primary treatment of orbital RMS includes Vincristine, Actinomycin-D and Cyclophosphamide (VAC) or VA chemotherapy. Local radiation therapy should be given after 12 weeks of chemotherapy.

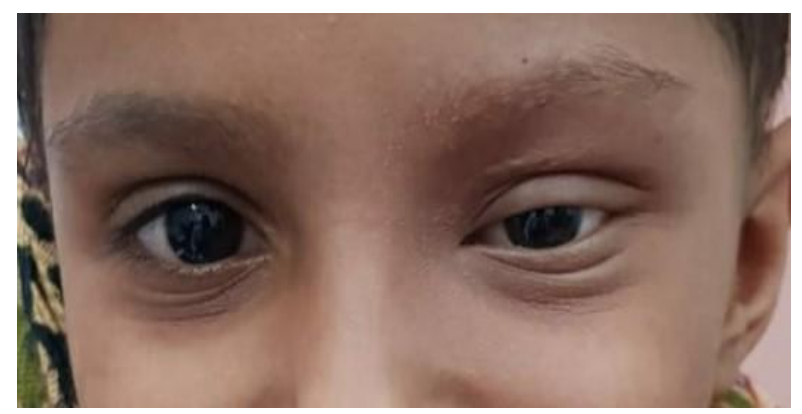

Fig.4 Patient had shown excellent response to chemoradiation

Technique of radiation therapy is important for minimizing corneal and lacrimal gland dose and also for preservation of useful vision. We had planned the radiation with IMRT technique. We had planned for $45 \mathrm{~Gy} / 25 \#$ at $1.8 \mathrm{~Gy} / \#$, but during treatment in 11\#, patient had shown excellent response to the treatment. Proptosis was reduced due to tumor shrinkage. So we had made new adaptive IMRT plan according to the regressed tumor to minimize the dose to adjacent OARs. Then patient was treated upto 45Gy with new adaptive RT plan.

Due to changes in tumor volume, patient's anatomy may change. Adaptive radiation aims to correct the morphological variations by making one or more plans during the treatment course. Imaging is used to detect the variations and change the treatment plan accordingly. At present, adaptive radiation is used for head and neck cancers. But in our patient, we had done adaptive radiation planning due to excellent response of tumor to the treatment. 


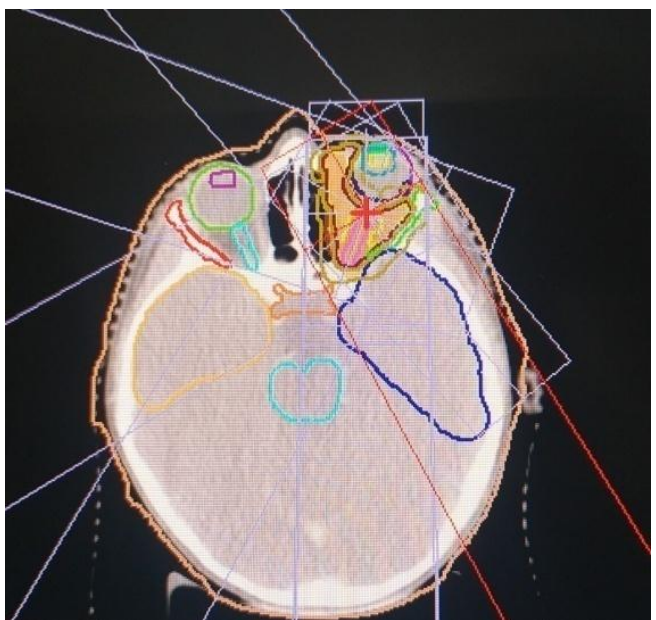

Fig. 5 Conformal IMRT planning

In orbital RMS, no surgical procedure should be used that may compromise vision or loss of function. Orbital exentration should be reserved for salvage treatment.

Follow up of patient should be done at 3 month interval with good ocular examination. At present, our patient is kept under surveillance with normal vision in both the eyes.

\section{Conclusion}

Orbital RMS carries favorable prognosis with good survival rates, so accurate diagnosis and treatment is important. Adaptive Radiation treatment in Orbital RMS is good option to minimize dose to normal adjacent structures but further studies and evaluation is needed in this aspect.

\section{Acknowledgment}

The authors want to thanks to patient parents for giving their all information regarding medical reports.

\section{Conflicts of interest}

There are no conflicts of interest.

\section{References}

1. Keziah N. Malu, Joseph A. Ngbea, Hameed Mohammand. Primary orbital rhabdomyosarcoma: a case report
2. Rajnish Nagarkar, Shirsendu Roy, Mohammad Akheel, Vijay Palwe, Nayana Kulkarni. Rhabdomyosarcoma of orbit: A case report and review

3. D.H. Abramson, R.M. Ellsworth, P. Tretter, J.A. Wolff, F.D. Kitchin. The treatment of orbital rhabdomyosarcoma with irradiation and chemotherapy.

4. R.H. Sagerman, P. Tretter, R.M. Ellsworth. The treatment of orbital rhabdomyosarcoma of children with primary radiation therapy.

5. Xin Ge, Dong-Sheng Haung, Ji-Tong Shi, Jian-Min Ma. Multidisciplinary collaborative therapy for 30 children with orbital rhabdomyosarcoma.

6. ShrikantBalasaheb Mali. Adaptive radiotherapy for head and neck cancer. 